\title{
Gradhiva
}

GRADHI

Revue d'anthropologie et d'histoire des arts

$1 \mid 2005$

Haïti et l'anthropologie

\section{Des comparables dans le champ du politique}

Entre nous, ethnologues et historiens

\section{Marcel Detienne}

\section{(2) OpenEdition}

\section{Journals}

Édition électronique

URL : http://journals.openedition.org/gradhiva/69

DOI : $10.4000 /$ gradhiva.69

ISSN : 1760-849X

Éditeur

Musée du quai Branly Jacques Chirac

\section{Édition imprimée}

Date de publication : 1 mai 2005

Pagination : 5-17

ISBN : 2-915133-08-5

ISSN : 0764-8928

Référence électronique

Marcel Detienne, «Des comparables dans le champ du politique », Gradhiva [En ligne], 1 | 2005, mis en ligne le 10 décembre 2008, consulté le 21 septembre 2020. URL : http://journals.openedition.org/ gradhiva/69 ; DOl : https://doi.org/10.4000/gradhiva.69

Ce document a été généré automatiquement le 21 septembre 2020.

(c) musée du quai Branly 


\section{Des comparables dans le champ du politique}

Entre nous, ethnologues et historiens*

\section{Marcel Detienne}

1 Votre invitation à présenter un sujet de comparatisme expérimental sous le signe de Robert Hertz me touche plus que je ne peux l'avouer. Entre ethnologues et historiens : j'y insiste aujourd'hui d'autant que, voici quinze ans déjà, l'on m'avait fait savoir qu'en vérité je n'étais ni l'un ni l'autre. Je n'avais pas répondu, engagé que j'étais dans la métamorphose de l'entredeux qu'exigeait la quête des comparables. Si je prends aujourd'hui la liberté d'un nous, ethnologues et historiens, c'est assurément parce que certains anthropologues, et Gérard Lenclud en premier, m'ont encouragé à en faire usage, et que nous, historiens et ethnologues de bonne volonté ${ }^{1}$, nous avons mené à bien une série d'enquêtes: sur « comment faire du territoire», sur ce que signifie «transcrire les mythologies »
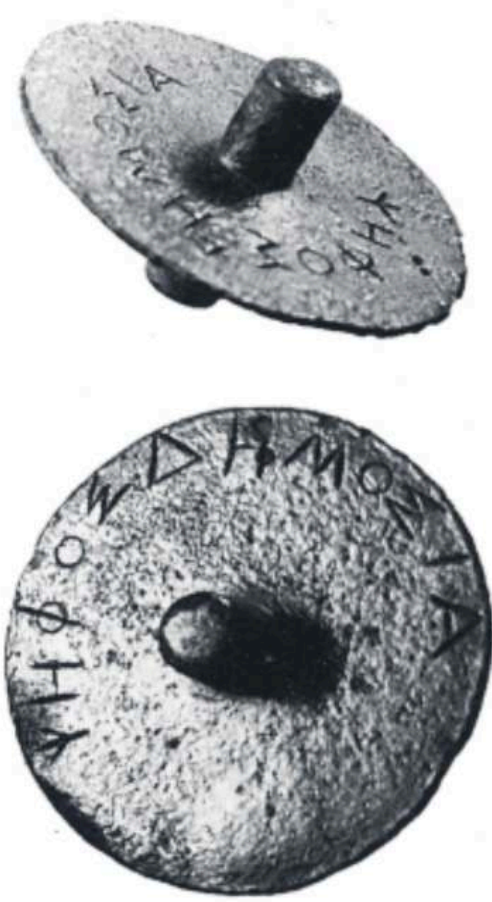
ou encore sur les «statuts des meurtriers et du meurtre $»^{2}$ entre les sociétés dites à histoire et d'autres étiquetées " sans histoire », par habitude. 
Dignitaires prenant la parole dans une assemblée, Ochollo, Éthiopie

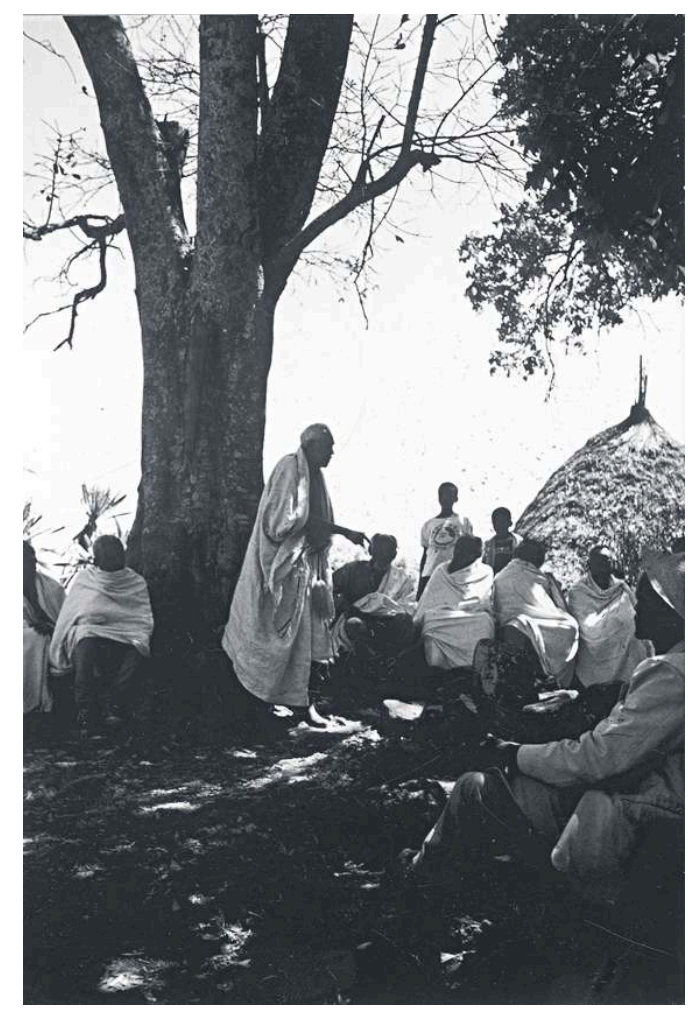

(c) Marc Abélès, droits réservés

\section{Les « sans histoire »}

Quand j'ai soumis à la gent intellectuelle quelques propositions sur l'art de construire des comparables, sous le titre sans ambiguïté de Comparer l'incomparable, ce sont des disciples de Marc Bloch qui ont les premiers ouvert le débat, en interrogeant «l'exercice de la comparaison, au plus proche, à distance " ${ }^{3}$. Les ethnologues, tout en reconnaissant à mi-voix que le comparatisme ne leur est pas étranger n'ont pas encore, au-delà de la litote, engagé la discussion sur les «comparables» dans le champ de l'anthropologie. Il m'a semblé que leur timidité était due en grande part à une certaine marginalisation de leur milieu. Dans la vieille province d'Europe qu'est la France de 2003, le rapport de force, on ne le sait pas, est extrêmement brutal: plus de 8000 historiens dont $60 \%$, au moins, cultivent hardiment le pré carré de l'histoire de France, face ou le dos à 300-350 ethnologues.

Il n'est pas inutile d'avoir à l'esprit cette disproportion - dont il serait urgent de faire l'histoire et l'histoire comparée dans le monde européen - pour comprendre l'indifférence absolue des uns et des autres à une réflexion sur les effets prolongés d'un partage disciplinaire comme celui que j'évoquais : d'un côté, des « sociétés à histoire ", de l'autre, des «sociétés sans histoire ». Les ethnologues s'en souviennent encore, les historiens n'avaient pas de raisons pressantes d'y revenir. L'affaire mérite d'être rappelée.

4 Il était une fois des sociétés sauvages, des peuples dits primitifs. On les appelait ainsi entre 1880 et 1900 . Manière moins courtoise de désigner ceux qui devaient longtemps 
porter, en France et dans ses expositions universelles, l'étiquette de "peuples non civilisés ", ou encore de "sociétés sans écriture ". D'une désignation à l'autre - c'était dans les années 1950 -, les historiens suivis par les ethnologues semblaient partir d'une constatation de fait: les «sans écriture » ne pouvaient être que «sans civilisation ». Tout enfant scolarisé par la République, apprend, depuis l'âge de Jules Ferry que l'histoire, la science historique commence avec les documents enregistrés, les pièces dites d'archives, donc avec la mise en écrit et la maitrise de l'écriture. Les sociétés dites archaïques ou primitives, les centaines de cultures découvertes par les ethnographes et analysées par les anthropologues ne pouvaient être que des sociétés «sans histoire». Les Lumières l'avaient énoncé, le $\mathrm{xIX}^{\mathrm{e}}$ siècle en avait fait une certitude. Il n'est naturellement d'histoire que de sociétés civilisées et de leurs États anciens. L'historien en herbe dans l'Europe de l'euro trouve ainsi confirmation de ce qu'il sait intuitivement depuis la maternelle et la maison paternelle, s'il naît doté de l'une et de l'autre. N'est-ce pas l'évidence même? L'histoire, science et discipline, n'est concernée que par les sociétés de l'écriture. La « Nouvelle Histoire » et les différentes « New History » y sont restées fidèles, quelles que soient les orientations épistémologiques : connaissance des hommes dans le temps, savoir du changement, représentation du passé, ou devoir de mémoire, le soir après l'école. Ce n'est qu'un partage obsolète, mais l'héritage en est si pesant au pays de la sclérose disciplinaire que je n'ai pas encore réussi à mobiliser celles et ceux qui pourraient le mettre à mal.

\section{Profession, comparatiste}

5 Profession donc: comparatiste. Dans les États-Unis d'aujourd'hui, la formule ne surprend pas, elle est comme bien reçue, elle éveille la curiosité de l'officier d'immigration, la sympathie du voisin de campus et de mon dernier déménageur. Dans les États-Unis d'Europe, jusqu'à présent, la réaction est volontiers de perplexité, sinon de méfiance et vite agressive. Soupçon d'avoir affaire à un nouvel imposteur: un « comparatiste » de quoi ? en quoi ? pour quoi?

6 Travailler, penser à plusieurs, entre ethnologues et historiens : quoi de neuf, me dira-ton? Il y a belle lurette qu'ethnologues et historiens ont fait connaissance et qu'ils cheminent de conserve. En effet, ils naviguent en conservant à vue, c'est le sens d'aller de conserve. On s'observe, on se frôle, on s'emprunte, parfois, là un objet, aussitôt appelé «nouvel objet », ici une formule qui donne le frisson agréable si bien venu de penser autrement. Le beau "regard éloigné » de l'anthropologie trouble, il séduit l'Histoire, surtout si d'aventure, à son miroir, un beau matin, elle se trouve mauvaise mine, un peu moins belle, un peu plus épaissie. Entre les deux savoirs, il s'agit le plus souvent d'un flirt, rarement d'une histoire. Les plus sérieux sont déjà retournés à leurs affaires. Les historiens en profitent pour répéter qu'ils préfèrent comparer entre eux, oui, entre voisins proches et de vieille date. Les plus sages avouent même un faible pour les similitudes et les analogies. Dans ses livres testaments sur l'Identité de la France, Fernand Braudel y revient "avec bonheur" ${ }^{4}$. Soit. L'institution, le clergé, les Académies, tout ce qui compte sous le ciel déconseille vivement à l'Histoire de vivre en concubinage avec l'Ethnologie. Elle est aguichante, bien sûr, mais vraiment pas du même milieu. D'ailleurs, on la dit sans grand avenir. Aujourd'hui, au chômage ; demain, qui sait? Sans papiers. Mes aînés m'avaient averti : ce n'est pas ainsi qu'on peut espérer finir dans un fauteuil à l'Académie. 
7 Je persévère: profession? Comparatiste, oui et même expérimental et constructif. Expérimental, d'abord. En quel sens? Historiens et ethnologues ensemble, nous sommes riches du savoir de centaines, de milliers de cultures et de sociétés différentes, dans le temps et dans l'espace. Ma conviction profonde, c'est que notre tâche commune est d'analyser les sociétés humaines, de comprendre le plus grand nombre de leurs productions culturelles. Pourquoi ne pas « expérimenter » quand cela est possible et, de plus, excellent pour l'activité intellectuelle des uns et des autres? Cela suppose travailler ensemble, librement, pendant des années, en allant d'une société à l'autre, en compagnie, elle est indispensable, des gens de terrain, des experts, des spécialistes. Sans la volonté active d'un Entre-Nous, sans le petit laboratoire ambulant d'ethnologues et d'historiens, à chaque fois plus ou moins renouvelé, il ne peut y avoir de comparatisme expérimental et constructif.

\section{Des lieux du politique}

Du politique, ou des lieux du politique. Vous le savez mieux que moi, le sens commun aime à croire que le ou la politique est tombé(e) du ciel, un beau jour, et dans l'Athènes de Périclès sous la forme miraculeuse et authentifiée de la démocratie. Il va de soi que son histoire divinement linéaire nous conduit par la main depuis la révolution américaine et en passant par la Révolution ladite française jusqu'à nos chères sociétés occidentales, si heureusement convaincues que leur mission est de convertir les autres peuples à la vraie religion de la démocratie.

9 Je n'ai cessé de montrer le respect le plus filial pour la " gent helléniste " ${ }^{5}$ et c'est en partant de ce bon sentiment que je me suis préoccupé d'en savoir davantage sur quelques commencements parmi les centaines de petites cités qui surgissent entre le VIIIe et le $\mathrm{VI}^{\mathrm{e}}$ siècles, $\mathrm{BC}$ of course. Il me faut quand même dire, très confidentiellement, que d'un continent à l'autre - l'hellénisme est universel -, des historiens à rosette, des Immortelles à Coupole, et des savants à l'autorité strictement germanique disputent avec autant de courtoisie que de pugnacité, de quoi ? de l'invention du politique en Grèce, bien sûr, mais surtout de sa date de naissance, lieu, jour, heure, de la couleur de ses yeux, de la nature de son sexe, question majeure sur nos campus, "le politique " (en français), tò politikon étant un faux neutre hérité du grec où semble l'avoir glissé un conférencier venu d'Halicarnasse à Athènes, un certain Hérodote.

À peine avais-je pris connaissance de l'état de la question que, par mesure d'hygiène, je me mettais en congé de la cité grecque, sans perdre temps et papier à inventorier les habits de confection qui lui ont été réservés à Munich, dans le Quartier Latin, à Cambridge pour ne citer que les plus cotées des maisons de la Haute Couture. Comme tout un chacun, il m'est arrivé de rencontrer des gens affirmant sur la voie publique que toute société est politique, que le politique, c'est le pouvoir, ou que tout commence toujours avec la distinction de l'ami et de l'ennemi. Rien là qui ne m'ait détourné de faire le vide ou presque. En un mot de récuser toute définition préalable «du » politique. 


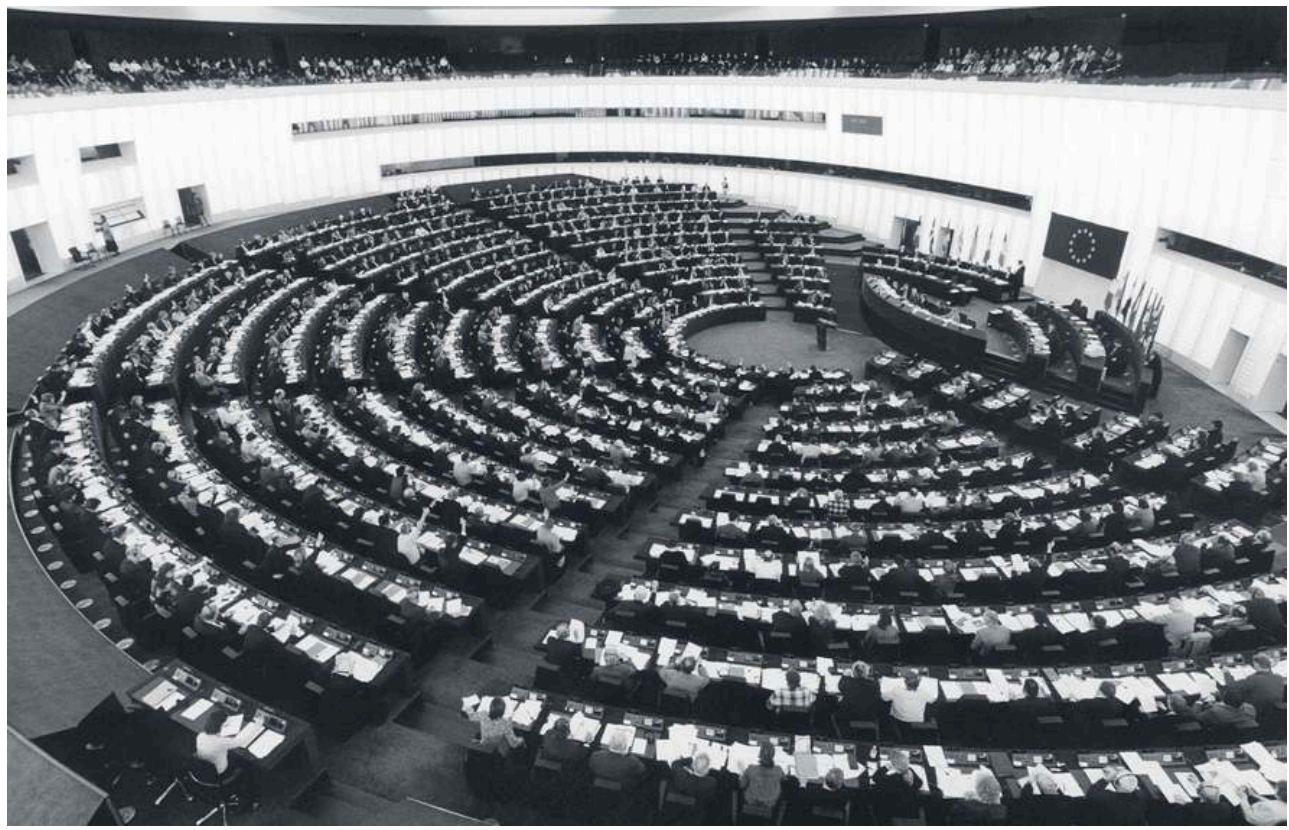

(C) Parlement européen

\section{Du vouloir s'assembler}

Faire le vide, mais jusqu'où? Jusqu'à des pratiques, des pratiques du s'assembler, des pratiques observées dans des situations de commencements, donc, si possible, sous des formes simples. Expliquons-nous. Des pratiques d'assemblée et, plus précisément, du vouloir s'assembler. Première orientation : vouloir s'assembler, soit, mais pourquoi ? pour débattre des affaires communes. Deuxième orientation et qui n'est pas innocente, car je suis amené à faire un choix très ciblé. Il ne s'agit pas de s'assembler pour une partie de pêche, ni pour échanger des plumes contre des griffes. Je sais combien les frontières qui semblent les plus sûres peuvent être poreuses et flottantes, mais les procédures de paroles tendues vers une idée de "ce qui est commun » délimitent peut-être un seuil provisoire de ce vouloir s'assembler où peut se fabriquer quelque chose comme du politique, voire un « lieu du politique ».

Dans le choix circonspect de "vouloir s'assembler pour débattre des affaires communes », il y a, me semble-t-il, un premier avantage : c'est de partir d'une catégorie souple sans être trop fluide. Il ne s'agit pas d'un paradigme commun comme, par exemple, celui de l'« humanisme civique ", proposé par John Pocock, ce vivere civile, un dispositif avec le Prince comme agent politique, et des associés, cousus main à la florentine, le citoyen, le rhétoricien et le législateur inspiré. Excellente entrée, à coup sûr, pour le monde anglo-saxon post-xvi ${ }^{\mathrm{e}}$ siècle mais aussi peu exportable que la catégorie « empire ». Les comparatistes qui se méfient des « homologies» doivent fuir comme la peste les « entrées d'encyclopédies» dites des Sciences Sociales. J'en appelle à l'observateur impartial, il existe encore : le vouloir s'assembler avec la couleur choisie n'est ni une catégorie trop locale, ni une notion trop générale, du genre «attrapetout ». 
13 La catégorie retenue pour l'enquête s'ouvre donc très directement sur une série de questions relatives à des pratiques concrètes. Qui met en branle le procès d'un s'assembler? Est-ce n'importe qui du groupe? Un ancien, un homme d'autorité, un chef élu? Un individu doté de pouvoirs religieux? Où se tient l'assemblée ? Dans un lieu qui n'est jamais le même? Ou dans un espace balisé, un emplacement fixe, aménagé, voire architecturé ? Est-ce un lieu ritualisé ? Discrètement ? Solennellement ? Qui ouvre une telle assemblée ? Comment se ferme-t-elle? Qui la préside et comment? Est-elle précédée ou non par un conseil restreint ? Et de quel type ? Y a-t-il comme un ordre du jour? Comment se fait la prise de parole? Selon quelle gestualité ? S'il y a débat, de quelle nature est-il ? Avec ou sans «contradiction»? Et le calendrier des assemblées, quel est son rythme? Quelle est la respiration de ce vouloir s'assembler? Y a-t-il une décision finale ? Par acclamation ? Vote à main levée ? Secret ? À la majorité ? Quel est le statut de la minorité ? Qu'en est-il d'un quorum, de son rapport à la totalité d'une assemblée et d'une communauté ?

14 À mesure que chemine l'approche complice entre ethnologues et historiens, plus les questions s'affinent, plus les différences prolifèrent au bénéfice de l'expérimentation. On le voit, il ne s'agit de rien d'autre que de faire réfléchir sur la complexité de la fabrique de quelque chose qui pourrait être « du politique ».

15 Autre avantage de l'entrée "vouloir s'assembler » et de ses mini-pratiques, c'est de permettre aisément la mise en perspective d'une série de sociétés aussi différentes et grandement éloignées entre elles que les communes italiennes du Moyen Âge européen, les monastères bouddhistes du Japon, les Constituants français, les Cosaques contemporains du Prince de Machiavel, les Ochollo d'Éthiopie aujourd'hui, les Circassiens du siècle dernier, les Sénoufos de Côte-d'Ivoire, les chanoines séculiers, bien fourrés, gros et gras de l'Occident moyenâgeux, les minuscules cités de la Grande Grèce et de Sicile, bref la vingtaine de cultures mobilisées au cours de la première étape d'une exploration à plusieurs ${ }^{6}$. Vingt sociétés, non pas en général, mais en microconfigurations analysées par celles et ceux qui les étudient de l'intérieur, souvent depuis vingt ans.

16 Il faut le redire : je n'ai jamais cessé d'apprendre en leur compagnie, et d'abord qu'il fallait débarrasser sa table de travail des lourds bibelots kitsch comme État ou Démocratie, en vente dans les supermarchés de Sciences humaines. Ensuite, se purger, matin et soir, de tout fantasme d'origine. Oublions, même s'il nous en coûte, la horde primordiale mise en images dans nos films préférés. Laissons dans le placard la sainte famille de notre enfance occidentale, sinon indo-aryenne. Mettons fin au règne de la "Commune", cette matrice crépusculaire née directement de la nature ou, pire, « sortie des mains de Dieu », aimait à dire Tocqueville, oui Tocqueville.

\section{Comparer des commencements}

Partir de formes simples, observer des pratiques de commencements, travailler sur des micro-configurations, car elles sont plus faciles à comparer en comparables que les États complexes ou semi-complexes, engoncés dans leurs macro-configurations. Certes, les commencements sont multiples et ne se ressemblent pas. Ils peuvent se faire tantôt dans un espace quasiment vide, sur table rase ou sur socle arasé, tantôt dans des contextes hautement sophistiqués. Le mouvement de naissance de ce que nous pourrions appeler un « lieu du politique » n'est jamais le même, d'une société à l'autre. 
Pour les premiers Cosaques, hommes libres autoproclamés, il y a d'abord la steppe et son silence de glace. Pour tant de cités lilliputiennes dessinées sur le sable de la Grande Grèce, la terre est d'abord vierge et comme inoccupée, du moins à première vue. Pour les Pisans, révolutionnaires de la Commune marine en 1080, il y a, au contraire, la ville, ses nobles, les puissances de l'Empire et celles si proches de l'Église. Devant les Constituants français en quête d'un cercle voilant son hémicycle, il y a ce qui va bientôt être appelé l'Ancien Régime, le Roi et une société d'ordres et de privilèges à démanteler, à briser à coups de marteau.

Gardons l'œil sur les Constituants, ces mutants de 1789. Voilà un commencement fascinant, bien observable et qui, à partir de son formidable "vouloir s'assembler " pour débattre des affaires de tous (la formule ici fait mouche) invente avec fureur une série de pratiques d'assemblées, imagine un espace inédit pour accueillir des délibérations permanentes entre des "représentants de la Nation", disposant en principe d'un droit de parole égal pour tout ce qui concerne le Bien du Peuple, un Peuple bientôt sacré Souverain. En même temps que de multiples pratiques se déploient une réflexion à plusieurs voix sur le lieu inédit d'un politique qui récuse tout précédent. Vertu des commencements qui font voir comment des configurations s'ébauchent, quels éléments s'agencent pour donner forme à l'idée d'une communauté, aux modalités d'une sorte de souveraineté du groupe sur soi, à l'architecture mentale d'un espace public, à l'esquisse d'un type de citoyenneté.

\section{Comparables en vue}

19 Comparer au plus loin, c'est un jeu intellectuel avec le plaisir d'expérimenter à plusieurs et sans hâte. Pour en donner un aperçu, j'aimerais indiquer à vol d'oiseau quelques-uns des comparables possibles dans le vaste domaine du « Qui veut prendre la parole?", selon le titre choisi pour une première enquête et en écho à la formule du héraut ouvrant l'assemblée dans une cité grecque. L'opération la plus simple pour ce faire consiste à monnayer une série de notions qui semblent être de bons réactifs dans le champ du « vouloir s'assembler » à des fins bien délimitées. Je retiendrai trois de ces notions: celle de "chose publique» ou d'affaires communes; ensuite, celle de "citoyenneté », à mettre entre guillemets doubles; enfin, le couple de «similitudeégalité ».

\section{De la chose publique}

Privilégier des manières concrètes de s'assembler, c'est se donner l'occasion d'observer comment des représentations des affaires communes peuvent se façonner à travers les pratiques d'un vouloir s'assembler local. Vouloir : deux mots pour y insister. Des gens s'attroupent, d'autres sont rassemblés. Un chef, un roitelet peut ordonner un rassemblement; un attroupement se fait à l'occasion d'un incident, autour d'un accident, de ce qui surprend ceux qui passent par là. Circulez, il n'y a rien à voir. Vouloir s'assembler à des fins précises n'est jamais que l'œuvre d'une minorité, et qui doit être active. Qu'est-ce qui peut donc la motiver? Nous pourrions reposer la question à propos de certains types d'homme, plus porteurs que d'autres, sans vouloir en attendre une explication. 
21 J'ai choisi la formule du héraut de l'Antiquité non parce qu'elle est grecque, nul ne peut me soupçonner d'hellénomanie, mais parce qu'elle introduit un "vouloir » sans lequel ce politique-là, un parmi d'autres orienté vers des affaires communes à débattre, ne peut ni s'instituer, ni grandir et se déployer. Étrangeté d'un lieu réservé pour débattre d'affaires, communes à des individus naturellement différents et spontanément inégaux. Le "vouloir s'assembler» semble s'imposer progressivement à travers des pratiques et une sorte de mise en scène qui va faire découvrir à la collectivité quelque chose comme une première souveraineté du groupe sur soi. Débattre des affaires dites "de tous", parler de ce qui peut être senti et reconnu comme le plus essentiel au groupe, c'est pour la collectivité engagée dans ce travail, et il est considérable, se donner des représentations nouvelles à travers des pratiques spécifiques et des symbolismes convergents.

Il n'est rien de plus chétif qu'un lieu du politique ou un lieu d'égalité qui advient. Les chanoines séculiers, si je prends une collectivité au hasard, sont appelés à débattre trois fois par semaine des affaires communes. Ils élaborent un système de rémunération équilibrée pour compenser de fortes inégalités entre eux. Mais l'Universitas, ainsi s'appelle « la chose commune » des clercs séculiers, s'affirme avec le choix d'un lieu de réunion central comme peut l'être, pour les citadins et les bourgeois, le beffroi ou la halle. L'Universitas des chanoines possède ainsi un coffre destiné aux archives et un sceau qui lui confère une part d'autorité. Les Cosaques, Zaporogues ou ceux du Don, ne se réunissent certainement pas deux ou trois fois par semaine, mais ils pratiquent une égalité beaucoup plus stricte et qui couvre initialement tous les domaines d'activité : guerre, chasse, pêche, terres cultivées. La "Communauté » (Tovaristo, en ukrainien) est présente, elle existe non seulement quand tous les Cosaques forment sur la grande place un cercle large de plusieurs rangs, mais lorsque sont déposés au centre de l'assemblée la Masse du Chef de guerre, le Sceau des Juges et le Grand Encrier d'argent du Secrétaire. Pour penser et symboliser «Ce qui est Commun", les premières cités grecques ont imaginé le Foyer Public sous les traits d'Hestia, le Foyer Commun répondant à la Vesta des Romains. Il incarne l'idée de la Cité unitaire dans le lieu où, chaque jour, se réunissent les magistrats en charge des affaires communes. Dans le Japon médiéval, les réunions des moines bouddhistes empruntent une autre voie. L'assemblée est précédée d'un serment d'union ou d'harmonie, chacun s'oblige à tout faire connaître à l'ensemble de la communauté, ce qui concerne "chacun et les autres ", et l'assemblée qui se veut unanime considère que sa décision ou ses jugements se font sous le regard des dieux. Pour les moines, leurs arrêts ont une force plus grande que les volontés de la Cour impériale. Au pays Sénoufo, en Côte-d'Ivoire, au cœur de l'espace réservé à l'assemblée égalitaire des initiés, il y a une place vide, celle de la "Vieille », la place d'où s'énonce la parole de tous. En France, Claude-Nicolas Ledoux, architecte visionnaire, au temps de la Révolution, voulait loger l'Autel de la Patrie avec le Mourir pour Elle - au cœur de l'hémicycle de l'Assemblée nationale, NationPatrie, et voilà un lieu du politique à la limite de la surcharge.

\section{Et de la justice}

Chemin faisant, entre des cultures si heureusement contrastées, il m'a semblé que la catégorie "Justice» ne pouvait être incongrue dans la formation de ce que nous appellerons donc un «lieu du politique». Oublions le village d'où nous sommes 
originaires et où la séparation des pouvoirs fait partie du paysage natal. Peut-il y avoir des lieux d'égalité ou des lieux du politique sans que soit requis comme l'institution d'une justice? Une justice pour ceux qui, en ce lieu, considèrent qu'ils sont « semblables " par certains traits et davantage «égaux » que soumis les uns aux autres ou liés entre eux par un système de parenté et d'obligations inégales. Par des voies différentes, des sociétés qui s'ignorent peuvent découvrir qu'un rapport de justice entre égaux et semblables n'est pas indifférent à la constitution du lien politique. Plus simplement, il semble qu'une collectivité visant à fonder une sorte de souveraineté sur elle-même tend à se donner l'autorité de statuer sur le juste et l'injuste. Il lui faut fonder des tribunaux, choisir des juges, créer des jurys, car une des premières tâches d'une assemblée délibérante et exécutive ce pourrait être de mettre en place un ou des tribunaux du sang. Quel sang? Celui d'un nouveau « citoyen » ou le sang versé par un des membres de la communauté, ou encore par un étranger, voire des "gens du pays » dans l'espace que la nouvelle collectivité a fait sien et considère comme le terroir sur lequel la communauté exerce son autorité. Il semble qu'un homicide dans ces conditions porte directement atteinte à la Communauté en soi. C'est elle qui va se constituer en assemblée de jugement, ouvrant un espace où s'assemblent tous les " citoyens ", formant ainsi ce que nous appelons des jurys populaires. Une des actions les plus décisives pour les affaires communes et pour établir solidement la première Cité, c'est peut-être d'inviter tous les citoyens à constituer un seul et même jury. À Philadelphie, au cours de son voyage d'Observateur en Amérique, Tocqueville dit son étonnement devant l'invention admirable des jurys populaires, car cela veut dire, à ses yeux, que le plus grand nombre peut établir la vérité dans une affaire publique. Question qui lui semble celle-là même du droit de suffrage dans l'ordre politique qui se cherche au milieu du XIXe siècle de notre ère. Pour les Cosaques, ces hommes libres, ces guerriers, le Sceau de Justice fait partie de la triade symbolique par laquelle la Communauté se rend présente et se reconnaît elle-même. Dans le monde des communes italiennes, notaires et juristes vont très tôt rendre la justice pour les affaires de l'Universitas. En Italie du Nord, et par exemple à Milan, me dit-on, l'espace propre à la justice des «citoyens" est souvent plus ancien que celui des assemblées délibératives.

Permettez-moi d'en dire davantage : l'exercice d'une justice propre à la Commune ou à la Cité contribue fortement à l'autonomie des Affaires communes. Plus précisément, la justice peut aider à dégager certains traits de l'individu dans le cercle de sa " citoyenneté ». Avec l'avènement de la responsabilité criminelle, l'individu apprend à s'émanciper de la solidarité familiale; il tend à devenir un sujet de droit dans l'espace des tribunaux où peut se déployer avec l'accusation publique la défense argumentée. Un droit prend alors forme qui distingue des degrés de responsabilité et fixe un tarif des peines. Pour «qui prend la parole », pour le devenir "politique » du citoyen d'un lieu, c'est, je crois, une étape importante en direction de quelque chose comme un individu équitable, c'est-à-dire appelé à juger équitablement ses "égaux » et ses «semblables».

\section{De la citoyenneté en ébauches}

Deuxième notion qui se prête également à être monnayée : la " citoyenneté ", excellent réactif dans le champ des possibles du politique. Il suffit de se demander, par exemple, 
quelles sont les qualités souhaitées ou requises d'un de ceux qui veulent si obstinément s'assembler et pour parler exclusivement des affaires communes. Dans le cercle du «Qui veut prendre la parole?» Qui est ce "premier orateur»? Que doit-il être? Que doit-il avoir ? Comment se qualifie-t-il ? Le « droit égal » de la citoyenneté décrété, par exemple, par la Révolution française n'a pas de sens en dehors du contexte philosophique du XvIII ${ }^{e}$ siècle. Mais en quoi consiste concrètement le "droit à l'égalité " quand est proclamée et publiée la "Déclaration des droits de l'Homme», sur fond de dissolution d'un régime des Ordres avec ses privilèges en hiérarchie ? Vingt millions de citoyens d'un jour à l'autre ne signifient pas que surgissent de terre des citoyens actifs, engagés à tous les niveaux de la Nation dans la participation aux affaires publiques. En 1789, il suffit d'être " né en France " pour avoir le droit théorique à l'égalité, mais tout reste à faire pour les militants des assemblées primaires ou de sections. Prenons les groupes de deux cents ou cinq cents Grecs comme ceux qui vont s'établir, aux viIIe et $\mathrm{VII}^{\mathrm{e}}$ siècles avant notre ère, sur les bords de mer entre la Sicile et la mer Noire : leur "citoyenneté » en puissance commence avec le tracé d'un cercle, appelé agora assemblée -, à moins que ce ne soit avec le tirage au sort d'un lot de terre avant le départ ou sur le navire même. Chacun, en possession de ses armes, semble avoir déjà un droit égal dans les débats et dans les sacrifices alimentaires accomplis par la collectivité. Participer, avoir part, et part égale, à tout ce qui est du ressort du "Commun » ou de la Cité (polis), c'est le cœur battant d'une première citoyenneté centrée sur l'espace fixe réservé aux assemblées, aux débats publics, à la lutte des discours sur les affaires communes du groupe à peine installé.

C'est dans ces lieux neufs d'égalité et peut-être «du politique » que s'observe le mieux comment peuvent s'agencer les éléments constituants d'une « citoyenneté » focalisant sur « les affaires communes ». Être du pays et habiter sur un lopin de terre ne suffit pas, il faut faire partie du cercle de l'assemblée volontaire et prendre part à la justice, autre cercle ou cercle dans le cercle. Comment veulent et peuvent agir ensemble des "citoyens"? Les nations d'hier et leurs expérimentations successives sont ici très utiles pour donner forme à des types de citoyenneté, et donc aux critères qui vont permettre de distinguer les "citoyens" des étrangers de passage ou de résidence, d'établir une suite de gradations entre ceux que l'on accueille, ceux qui peuvent être intégrés et "naturalisés ", là aussi à des degrés divers, comme avoir ou non accès à des responsabilités publiques, aux plus hautes magistratures, ou encore à des fonctions essentielles à la cité ou au groupe. Tôt ou tard, l'Assemblée - Universitas ou Community attendra du citoyen actif qu'il soit doté de certaines qualités, avant d'exiger, cela s'est $v u$, qu'il possède des vertus spécifiques, celles-là peut-être qui vont orienter de manière décisive une Respublica ou son équivalent. 
Place d'assemblée à Ochollo, Éthiopie

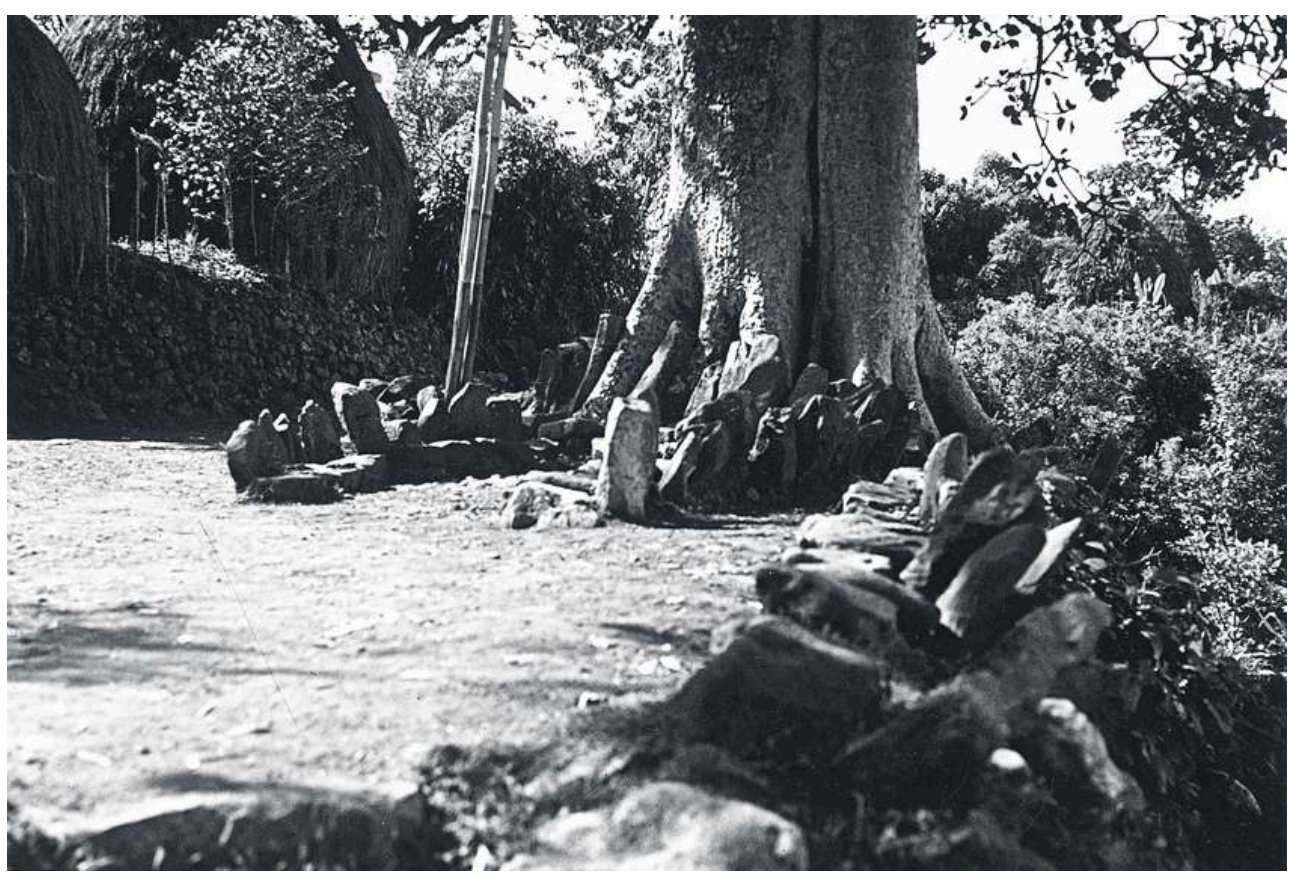

(c) Marc Abélès, droits réservés

\section{Semblables et égaux}

Troisième entrée ou notion, bonne à faire penser : "Similitude - Égalité ». On peut faire l'hypothèse que «vouloir s'assembler pour délibérer des affaires communes » présuppose que chacun s'accorde à reconnaître en l'autre du même cercle une similitude ou une forme d'égalité. La première analogie venue ne crée pas une similitude suffisante pour entrevoir la possibilité d'une «chose commune ». Rousseau pouvait croire que la citoyenneté des Anciens était inspirée par une sensibilité commune qui naissait de la familiarité et de la compassion entre membres d'une même petite patrie. En ce temps-là la volonté générale coulait de source. La similitude des sentiments et des opinions n'est sûrement pas la seule voie possible. Les Cosaques qui ont en commun de se vouloir "hommes libres " au milieu des princes et des serfs s'appellent entre eux «frères ». Leur père est le chef qu'ils se choisissent chaque année quand s'assemblent tous les "frères ", à la fois "semblables » et "égaux " par leurs pratiques d'égalité. Au départ, les Cosaques sont tous des hommes libres, comme les acteurs de la première commune de Pise au $\mathrm{XI}^{\mathrm{e}}$ siècle sont pour la plupart des marins libres mais qui peinent en mer de la même façon. Tandis que d'autres sont moines bouddhistes ou chanoines séculiers.

Il y a des sociétés où la similitude et l'égalité sont énoncées de manière explicite, à un certain état $d u$ "vouloir s'assembler». Par exemple, en Grèce ancienne, dans les minuscules cités de l'époque archaïque : chacun reçoit des droits égaux et part égale aux privilèges de la "citoyenneté ». Pareille similitude suppose que soient mis entre parenthèses les traits distinctifs de gens que différencient par ailleurs des statuts sociaux ou des rapports de parenté. Se reconnaître réciproquement des formes de similitude peut contribuer à faire exister l'idée de Communauté ou de Cité. Les 
pratiques d'égalité, quant à elles, ont une vertu constituante : égalité arithmétique d'abord, que ce soit dans le partage des terres, la répartition du butin ou la distribution des nourritures, par exemple, en parts égales et tirées au sort. L'égalité géométrique fait vite son chemin, avec ses différentes formulations. Reste que l'égalité, une fois découverte par un biais ou par un autre, peut devenir un cri de guerre, comme par exemple, à la fin du vie siècle, à Athènes. C'est le mot isonomie, "avoir une part égale ". De quoi ? De tout, bien sûr, sans que cela signifie toujours, comme à l'occasion de tant de guerres civiles, l'abolition des dettes et la redistribution des terres. Tandis que les Bostoniens que Tocqueville rencontre en 1831 se satisfont d'être égaux en droits, mais devant Dieu qui voit tout cela de fort loin. L'égalité, cela doit s'expliciter, se débattre en public, et le plus souvent se conquérir à chaque étape et à tous les niveaux du s'assembler. Une égalité théorique peut certes faire découvrir les vertus de la majorité pour les décisions de l'assemblée. Mais une égalité stricte articulée à des pratiques guerrières peut très bien s'accommoder de la non-reconnaissance d'une voix pour chacun des Semblables.

D'autres entrées nous guettent, à commencer par celle à double voie du public et du privé. Comment se façonne un espace public et qu'en est-il d'un privé qui se cherche sans hâte à la lisière entre des " citoyens " - par exemple, ceux qui se ressemblent et ont à faire à l'égalité - et des individus qui commencent, mais comment ? à différer par des traits singuliers ainsi que par leurs intérêts propres. Certes, il y a également la question, est-elle monnayable? des types d'homme, du moins ceux qui semblent à première vue plus porteurs que d'autres pour entrouvrir un lieu du politique ou pour ébaucher un espace égalitaire. Le guerrier, le moine, le marchand pour ne pointer que les plus visibles.

\section{Encore un mot}

Avertissement, si vous permettez. Dans ce comparatisme "entre nous », il n'est, il ne sera jamais question de juxtaposer une touche de Japon qui serait insensiblement tout le Japon, un zeste de Circassiens qui représenterait la masse du Caucase sur le champ et, pour la couleur, deux voire trois communes italiennes qui autoriseraient à écrire en conclusion d'un volume dans le genre "Comparative Studies", voici comment on s'assemble en Italie pour inventer le politique, tandis qu'au Japon...

31 Ce serait faire un long voyage de dupes pour reprendre le jeu de société de nos universités, où rien n'est plus excitant que de savoir si nos Athéniens ont vraiment connu la démocratie, la vraie bien sûr, ou encore, variante raffinée, si c'est Solon ou Clisthène à qui revient l'honneur à jamais d'avoir inventé le politique. Étrange singulier.

32 Je préfère croire que construire quelques comparables en nous livrant à une série d'expérimentations peut contribuer à faire réfléchir sur quelque chose comme du politique. Les politistes n'auraient-ils plus besoin d'espaces d'intelligibilité ? Un peu partout les historiens s'affairent à explorer les gésines de l'État, comme si le singulier devait régner absolument sur tout ce qui concerne l'Occident figé dans la grandeur de son origine incommensurable. deviendra un jour l'Italie, il y a plus de 70 communes, urbaines et rurales, avec archives 
et documents qui attendent les historiens curieux d'expériences d'un «Bon Gouvernement " ou d'une "Universitas ", souvent concomitantes et toujours neuves. Les mondes amérindiens, les communautés du Mexique contemporain, celles, innombrables, du continent africain offrent aux ethnologues, sociologues et historiens un vaste terrain d'expérimentation pour des approches comparatives. Oui, en quête d'autres et de nouveaux comparables7.

\section{NOTES}

*. Texte de la conférence Robert Hertz, prononcée à l'invitation de l'Association pour la recherche en anthropologie sociale (APRAS) le 26 juin 2002 à Paris.

1. Cf. le dossier « L'exercice de la comparaison », s. dir. de Lucette Valensi, Annales, HSS, 2002, 57 (1) : 27-144, revue dont le "comité de lecture» en sa clairvoyance a préféré écarter ma contribution ainsi que celle de Christian Jacob, en me proposant de commenter les analyses retenues pour la publication.

2. J'ai plaidé en ce sens dans Comparer l'incomparable, Paris, Le Seuil, 2000, et, plus allégrement dans "Murderous Identity. Anthropology History and the Art of Constructing Comparables ", Common Knowledge, 2002, 8 (1) : 178-187, ou dans «L'art de construire des comparables », Critique internationale, 2002, $14: 68-78$.

3. J'ai donné tous les textes à l'appui dans un petit livre : Comment être autochtone. Du pur Athénien au Français raciné, Paris, Le Seuil, 2003, en particulier, pp. 123-149 («Grandeur du Français raciné », fort utile, on en jugera, pour analyser le terreau qui donne à l'Extrême Droite de France ses belles couleurs).

4. J'ai donné tous les textes à l'appui dans un petit livre : Comment être autochtone. Du pur Athénien au Français raciné, Paris, Le Seuil, 2003, en particulier, pp. 123-149 («Grandeur du Français raciné ", fort utile, on en jugera, pour analyser le terreau qui donne à l'Extrême Droite de France ses belles couleurs).

5. Au point même d'essayer d'analyser certains blocages, fort improbables en milieu ethnologique. Qu'on me permette de signaler un mien article, généreusement reçu par la rédaction de L'Homme : «Rentrer au village : un tropisme de l'hellénisme? (cf. L'Homme, 2001, $157: 113-126)$.

6. Qui veut prendre la parole?, s. dir. de Marcel Detienne, Le Genre humain, 40-41, 2003, 433 p.

\section{INDEX}

Mots-clés : anthropologie, comparatisme, histoire, politique (le)

Keywords : anthropology, comparatism, history, politicism 
AUTEUR

\section{MARCEL DETIENNE}

Johns Hopkins University, Centre Louis Marin, Department of Classics / Baltimore, MD, dyono@jhu.edu 